\title{
Osteopenia in cerebral palsy
}

\author{
N J Shaw, C P White, W D Fraser, L Rosenbloom
}

\begin{abstract}
The bone mineral density of the lumbar spine was assessed in nine non-ambulant children with cerebral palsy combined with measurements of serum 25hydroxyvitamin D, parathyroid hormone, and urinary calcium excretion. Three children with recurrent fractures received treatment with bisphosphonates for periods ranging from 12-18 months.

All the children demonstrated a severe reduction in bone mineral density even when allowance was made for their body weight. There were no consistent abnormalities of vitamin $D$ or parathyroid hormone status. Three children had gross hypercalciuria. Each of the children treated with bisphosphonates demonstrated an increment in bone density ranging from $20-40 \%$ with no apparent adverse effects.

(Arch Dis Child 1994; 71: 235-238)
\end{abstract}

It is well known that immobilisation predisposes to bone resorption, which can reduce bone mineral density leading to an increased risk of fractures. A group of children who are at particular risk from this problem are those with physical handicaps that render them immobile, such as those with severe cerebral palsy. Long bone fractures are not uncommon in these children and may lead to increased hospitalisation in this already vulnerable group. The availability of new antiresorptive drugs in the form of bisphosphonates offer a potential therapeutic option.

The intention of this study was to examine a group of children with severe cerebral palsy to determine the prevalence of osteopenia and to see if it was related to abnormalities of vitamin $\mathrm{D}$ or parathyroid hormone status. In addition the benefit of treatment with bisphosphonates was studied in a small group of children who were having recurrent fractures.

Health, Alder Hey

Children's Hospital, Liverpool

N J Shaw

Department of Neurology, Alder Hey Children's Hospital, Liverpool

C P White

L Rosenbloom

Department of Clinical Chemistry, Royal Liverpool University Hospital

W D Fraser

Correspondence to: Dr N J Shaw, Institute of Child Health, The Children's Hospital Ladywood Middleway, Birmingham B16 8ET.

Accepted 15 June 1994 one child was receiving anticonvulsants at the time of the study. Four children were receiving either phenytoin or phenobarbitone that are known to affect vitamin $\mathrm{D}$ metabolism in the liver. The bone mineral density of three lumbar vertebrae $\left(\mathrm{L}_{2}-\mathrm{L}_{4}\right)$ was determined in eight of the children by dual energy $x$ ray absorptiometry (DXA) using an Hologic QDR 1000 scanner. This has a radiation dose of 4 millirem and coefficient of variation of $2 \%$. The remaining child (patient 1) had a measurement of bone mineral density of $\mathrm{L}_{2}-\mathrm{L}_{4}$ determined using quantitative computed tomography (QCT) on a Siemens computed tomograph. This has a radiation dose of 100 millirem and a precision of 3\%. This was performed because of the lack of reference data available for a 2 year old using DXA. The mean value for bone mineral density in each child measured by DXA was converted into a SD score by comparison with the mean and SD values obtained in a group of healthy British children. ${ }^{1}$ This reference data was also obtained on an Hologic QDR 1000 scanner and a calibration phantom was used to ensure the two machines produced similar readings. SD score was also calculated for the child measured by QCT by comparison with published data in children. ${ }^{2}$ As bone mineral density measured by DXA is influenced by body size, ${ }^{3}$ comparison was made with reference data matched for body weight as well as for age.

Random non-fasting blood samples were obtained for measurement of calcium, phosphate, and alkaline phosphatase. Plasma calcium was measured using a colorimetric assay, plasma phosphate using a phosphomolybdate reagent, and alkaline phosphatase using a p-nitrophenylphosphate method. Each of these was measured using a Technicon RAXT analyser. Serum parathyroid hormone was measured with an immunoradiometric assay that recognises the intact molecule (Incstar Corp) and 25-hydroxyvitamin D using a straight phase high performance liquid chromatography method that enables resolution into the two main components 25-hydroxyvitamin $\mathrm{D}_{2}$ and 25-hydroxyvitamin $\mathrm{D}_{3}$. The coefficients of variation for the parathyroid hormone assay were $2.5 \%$ intra-assay and $6 \cdot 1 \%$ interassay at a concentration of $4 \cdot 2$ $\mathrm{pmol} / \mathrm{h}$ and $3 \cdot 1 \%$ and $5 \cdot 6 \%$ at a concentration of $31.5 \mathrm{pmol} / 1$. The coefficients of variation for the 25-hydroxyvitamin $\mathrm{D}_{2}$ assay were $10 \cdot 7 \%$ intra-assay and $14.3 \%$ interassay at a concentration of $7.5 \mathrm{ng} / \mathrm{ml}$, and for the 25-hydroxyvitamin $\mathrm{D}_{3}$ assay $8.3 \%$ and $11.3 \%$ at a concentration of $23 \mathrm{ng} / \mathrm{ml}$. Random urine samples were obtained in the non-fasting state for determination of calcium excretion by the calcium/creatinine ratio. The reference data 


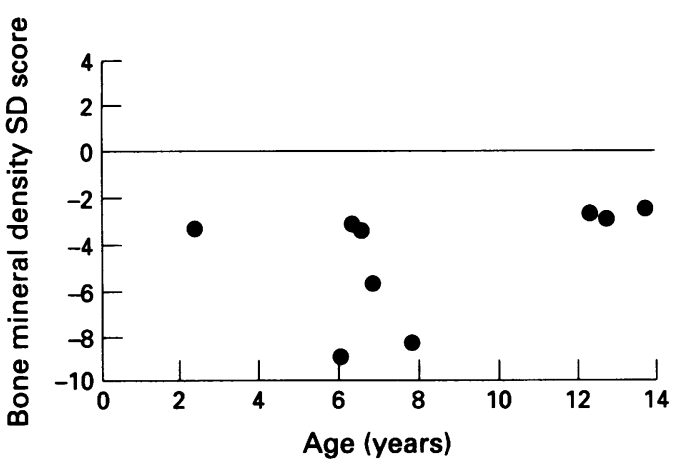

Figure 1 Bone mineral density of lumbar spine in comparison with age matched controls.

for parathyroid hormone and calcium excretion were those obtained in a group of healthy British children. ${ }^{4}$ The vitamin $\mathrm{D}$ reference ranges were those determined by the clinical chemistry department at the Royal Liverpool University Hospital.

Three children in the study who were having problems with recurrent long bone fractures, having at least two fractures in a six month period, were treated with bisphosphonates. One child aged 2 years was treated with disodium etidronate given via a nasogastric tube, on an empty stomach, in a dose of $7.5 \mathrm{mg} / \mathrm{kg}$ (total dose $100 \mathrm{mg}$ ) for two weeks in every three month cycle, in combination with daily vitamin $\mathrm{D}$ (400 IU) and calcium supplements (400 mg elemental calcium). The total duration of treatment was 18 months. The dose of disodium etidronate was based on the recommended adult dose of 5-10 $\mathrm{mg} / \mathrm{kg}$. The two other children, aged 6 and 13 years, were treated with disodium pamidronate in a dose of $0.4 \mathrm{mg} / \mathrm{kg}$ given as an intravenous infusion over two hours, every three months, for a 12 month period. The dose of disodium pamidronate was decided in relation to doses used in adults by one of the authors (WDF) as there is no published dosage for its use in osteoporosis. Repeat measurements of bone mineral density were undertaken during the course of their treatment in addition to routine measures of full blood count, plasma creatinine, calcium, phosphate, alkaline phosphatase, and urinary calcium excretion. Statistical analysis was performed by using Spearman's rank correlation coefficient to assess correlation.

Informed parental consent was obtained in each case and the study received approval

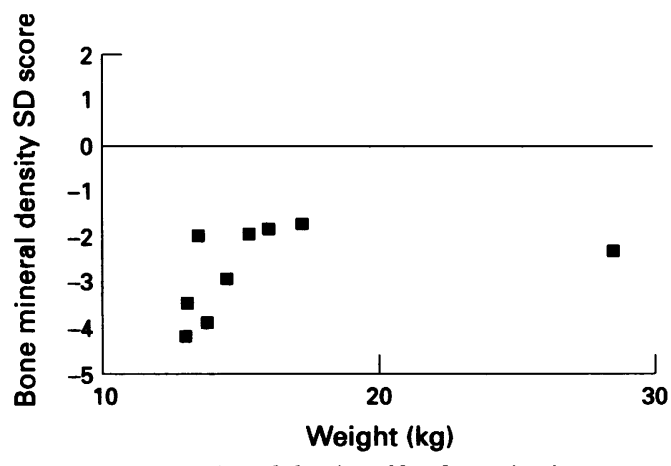

Figure 2 Bone mineral density of lumbar spine in comparison with body weight matched controls.
Table 1 Concentrations of 25-hydroxyvitamin $D_{2}$ and $D_{3}$ in nine children with cerebral palsy

\begin{tabular}{lll}
\hline $\begin{array}{l}\text { Patient } \\
\text { No }\end{array}$ & $\begin{array}{l}\text { 25-Hydroxyvitamin } D_{2} \\
(\text { ng/ml })\end{array}$ & $\begin{array}{l}\text { 25-Hydroxyvitamin } D_{3} \\
(\text { ng/ml) }\end{array}$ \\
\hline 1 & $<2$ & 27 \\
2 & $<2$ & 31 \\
3 & $<2$ & 42 \\
4 & $<3$ & 22 \\
5 & $<5$ & 20 \\
6 & $<3$ & 15 \\
7 & $<5$ & 25 \\
8 & $<2$ & 4 \\
9 & $<3$ & 11 \\
Normal range & $<10$ & $10-60$ (summer) \\
& & $5-25$ (winter) \\
\hline
\end{tabular}

from the Royal Liverpool Children's Hospital ethical committee.

\section{Results}

The bone mineral density SD scores using age and sex-matched control data are shown in fig 1. All the children studied had values more than 2 SDs below the mean ranging from $-2 \cdot 5$ to $-8 \cdot 9$. When adjustment is made for body weight by calculating SD scores using weight matched control data (fig 2), although there is an improvement in the degree of demineralisation, a severe reduction persisted with $\mathrm{SD}$ scores ranging from $-1 \cdot 7$ to $-4 \cdot 2$. The values for measurement of 25-hydroxyvitamin $\mathrm{D}_{2}$ and $\mathrm{D}_{3}$ are shown in table 1 . All but two children (patients 5 and 7) had concentrations of 25hydroxyvitamin $\mathrm{D}_{2}$ at the limit of detection of the assay. Concentrations of 25-hydroxyvitamin $\mathrm{D}_{3}$, however, were satisfactory in all but one child (patient 8 ). The values for the measurement of intact parathyroid hormone and the urine calcium/creatinine ratio are shown in table 2. Seven of the children had values for parathyroid hormone within the reference range with two children (patients 5 and 8) having raised values. The child with the highest concentration of $12.6 \mathrm{pmol} / \mathrm{l}$ was the same child who had the low concentration of 25-hydroxyvitamin $\mathrm{D}_{3}$, and was also hypocalcaemic. All the other children in the study had normal values for plasma calcium, phosphate, and creatinine. Alkaline phosphatase values were normal in all but one child (patient 1). Three children had evidence of gross hypercalciuria, the remainder having calcium/ creatinine ratios within the reference range.

No significant correlations were seen between the bone mineral density SD scores and vitamin $\mathrm{D}$ and parathyroid hormone status or urinary calcium excretion. There was a

Table 2 Concentrations of parathyroid hormone and urine calcium/creatinine ratio in nine children with cerebral palsy

\begin{tabular}{lcc}
\hline $\begin{array}{l}\text { Patient } \\
\text { No }\end{array}$ & $\begin{array}{l}\text { Parathyroid hormone } \\
\text { (pmoln) }\end{array}$ & $\begin{array}{l}\text { Calcium/creatinine } \\
\text { (mmol/mmol) }\end{array}$ \\
\hline 1 & 2.6 & 2.40 \\
2 & 1.0 & 5.50 \\
3 & $1 \cdot 1$ & 0.42 \\
4 & 3.4 & 0.18 \\
5 & 6.1 & 1.21 \\
6 & 2.3 & 0.07 \\
7 & 1.6 & 0.08 \\
8 & 12.6 & 0.03 \\
9 & 1.4 & 0.01 \\
Normal range & $1.1-3.5$ & $<0.70$ \\
\hline
\end{tabular}




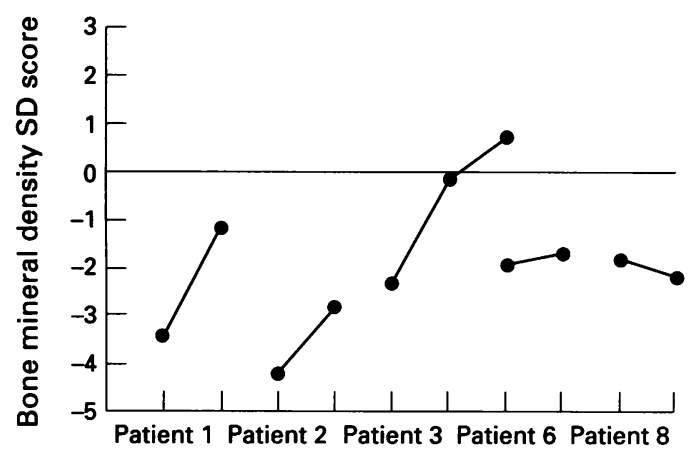

Figure 3 Changes in bone mineral density of lumbar spine in three children treated with bisphosphonates (patients 1 , 2, and 3) and two untreated children (patients 6 and 8).

strong correlation between bone mineral density and body weight $(r=0.83, p=0.01)$.

The changes in bone mineral density SD scores in the three children treated with bisphosphonates are shown in fig 3. Patient 1, who was treated with disodium etidronate, showed an improvement of bone mineral density SD score from -3.4 to -1.2 over an eight month period representing an overall $40 \%$ increment. Patient 2 who was treated with disodium pamidronate showed a change in bone mineral density SD score from $-4 \cdot 2$ to -2.8 over a 10 month period representing a $20 \%$ increment. Patient 3, who was also treated with disodium pamidronate, showed an initial change in bone mineral density SD score from -2.3 to -0.15 over a year with a further increase to $+0 \cdot 17,18$ months after the first scan representing an overall $23 \%$ increment. In comparison the change in bone mineral density SD score of two untreated patients (patients 6 and 8 ) is shown six months after the initial scan. Patient 6 showed a change in bone mineral density SD score from -1.92 to -1.66 , which is a $3 \%$ increment, while patient 8 showed a change in bone mineral density SD score from $-1 \cdot 8$ to $-2 \cdot 14$ representing a $3 \%$ decrease.

No adverse effects associated with the bisphosphonate treatment were seen during the time of administration. A progressive fall in plasma phosphate within the reference range was seen in the two children treated with disodium pamidronate, during the course of their treatment. The raised alkaline phosphatase in patient 1 before treatment normalised within six months of starting disodium etidronate with vitamin $\mathrm{D}$ and calcium supplementation. There was no reduction in the hypercalciuria seen in patient 1 during the treatment, but that seen in patient 2 normalised after the first infusion with disodium pamidronate.

\section{Discussion}

This study has demonstrated that osteopenia is a very common finding in children with severe cerebral palsy who are non-ambulant. This persisted despite correcting for body weight, as it is known that measurements of bone density using DXA are influenced by body size. ${ }^{3}$ The aetiology of this reduction in bone density in these children is likely to be multifactorial with important factors being immobilisation, poor nutritional status, and anticonvulsant use.

Osteopenia secondary to immobilisation is well recognised, having been described in diverse situations from spina bifida ${ }^{5}$ to space flight. ${ }^{6}$ Histological studies have shown an increase in bone resorption and a decrease in bone formation. In children with spina bifida, ${ }^{5}$ a relationship between ambulatory status and bone density has been demonstrated, consistent with the observation that weight bearing exercise is important for development of bone mass. ${ }^{7}$ In another recent study of bone density in children with cerebral palsy, ${ }^{8}$ which included ambulant as well as non-ambulant patients, higher bone density values were seen in the ambulant patients.

The results for 25-hydroxyvitamin D status in these children showed low values for 25hydroxyvitamin $\mathrm{D}_{2}$ (ergocalciferol) in many of the children. This is not unexpected as none of them were receiving vitamin $\mathrm{D}_{2}$ supplements. Concentrations of 25-hydroxyvitamin $\mathrm{D}_{3}$ (cholecalciferol) that reflect cutaneous production of vitamin $\mathrm{D}$ by sunlight exposure were satisfactory in all but one child. Thus these children are able to maintain satisfactory vitamin $\mathrm{D}$ status by sunlight exposure and there appears to be no strong indication for vitamin D supplementation of their diet.

It is well known that anticonvulsants, in particular phenytoin and phenobarbitone, have been associated with rickets and osteomalacia. ${ }^{9}$ This is due to induction of hepatic microsomal enzymes leading to increased catabolism of vitamin D. Low concentrations of 25hydroxyvitamin $\mathrm{D}$ have been demonstrated in some but not all patients. ${ }^{10}$ Anticonvulsant use has also been linked with inhibition of calcium absorption from the gut and impaired calcitonin secretion. ${ }^{11}$

In this study only one child receiving phenobarbitone had a subnormal concentration of 25-hydroxyvitamin $\mathrm{D}_{3}$, the other three children having values well within the reference range. Therefore vitamin $\mathrm{D}$ status did not appear to be relevant to the degree of osteopenia. The study by Root et al also found normal total 25-hydroxyvitamin D status and no relationship to bone density. ${ }^{8}$ Studies of calcium absorption were not undertaken as part of this study.

Similarly there was no consistent abnormality of parathyroid hormone secretion and no relationship to the degree of osteopenia. The high parathyroid hormone concentration seen in the child with vitamin $\mathrm{D}$ deficiency was likely to be due to secondary hyperparathyroidism as a consequence of the hypocalcaemia.

Gross hypercalciuria was seen in three of the children, this being present in two of the children with recurrent fractures and one other child who had not sustained a fracture. This is a recognised feature of immobilisation and occurs due to increased bone resorption. ${ }^{6}$ Although not performed in this study, such children should probably be screened for nephrocalcinosis using ultrasound.

The effect of bisphosphonate treatment on bone mineral density in the three children who 
sustained recurrent fractures was encouraging. The incremental increases seen were much more than could be accounted for by changes with body size with time. It is possible that puberty was a contributing factor for the increment in bone mineral density in the 13 year old girl but was not an influence in the other two patients. The changes were also significantly more than have been seen in adults treated for postmenopausal osteoporosis. ${ }^{12}$ Although there is extensive experience of their use in adults, there is very little experience to date in children. They are analogues of pyrophosphate and act by inhibiting osteoclastic mediated bone resorption. It has been suggested previously that they may have a role in preventing bone loss caused by immobilisation. ${ }^{5}$ The fall in plasma phosphate, seen in the two children treated with disodium pamidronate, is a recognised effect of its treatment due to secondary hyperparathyroidism decreasing renal tubular reabsorption of phosphate. ${ }^{13}$ As such it is a normal homoeostatic response rather than a side effect.

There have been some concerns, particularly with disodium etidronate, that inhibition of bone mineralisation may also occur but this appears to be when used in doses greater than $20 \mathrm{mg} / \mathrm{kg}$ or for prolonged periods. ${ }^{14}$ Histological studies in adults who had received low dose disodium etidronate in a cyclical manner for three years have not demonstrated mineralisation abnormalities. ${ }^{12}$ Some reservations must remain, however, about their use in children with growing and maturing bones. In this particular study they were used because of a difficult clinical situation to try and prevent recurrent fractures and hospital admissions.

The use of bisphosphonates in postmenopausal osteoporosis have been associated with a reduction in fracture rate and it is encouraging to report that only one of the three treated children had a further fracture in the year after the start of treatment. ${ }^{12}$ However, it is recognised that this study is small and these preliminary results would need to be followed by a placebo controlled randomised study. There is certainly no role at present for the use of bisphosphonates in children with radiological osteopenia in the absence of fractures.
There is currently no product licence for their use in children and their long term safety is unknown in this group. At present their use in children remains experimental.

In this particular group of children, an improvement in their nutritional status would probably help to improve their bone density and thus reduce their fracture risk. This would not, however, negate the effects of immobilisation and although they are unlikely to become mobile the role of increased weight bearing exercise should be considered as an additional preventative measure.

We are grateful to the nursing staff of $U$ ward at Alder Hey Children's Hospital for their assistance, the radiographers at the Women's Hospital in Liverpool for performing the DXA scan and Mike Davie and Mike Haddaway at the Robert Jones and Agnes Hunt Hospital in Oswestry for allowing us access to their reference data for bone mineral density.

1 Davie MWJ, Haddaway MJ. Bone mineral content and density in healthy subjects and in osteogenesis imperfecta. Arch Dis Child 1994; 70: 331-4.

2 Gilsanz V, Roe TF, Mora S, Costin G, Goodman WG Changes in vertebral bone density in black girls and white girls during childhood and puberty. $N$ Engl $f$ Med 1991; 325: $1597-600$.

3 Carter DR, Bouxsein ML, Marcus R. New approaches for interpreting projected bone densitometry data. $\mathcal{f}$ Bone interpreting projected bone

4 Shaw NJ, Wheeldon J, Brocklebank JT. Indices of intact serum parathyroid hormone and renal excretion of calcium, phosphate, and magnesium. Arch Dis Child 1990, 65: 1208-11.

5 Rosenstein BD, Greene WB, Herrington RT, Blum AS Bone density in myelomeningocoele: the effects of ambulatory status and other factors. Dev Med Child Neurol 1987; 29: 486-94.

6 Mazess RB, Whedon GD. Immobilization and bone. Calcif Tissue Int 1983; 35: 265-7.

7 Slemenda CW, Miller JZ, Hui SL, Reister TK, Johnston CC. Role of physical activity in the development of skeletal CC. Role of physical activity in the development of ske
mass in children. $\mathcal{I}$ Bone Miner Res 1991; 6: 1227-33.

8 Root L, Cervera PB, Glasser D. Gertner J, Pineda S. Bone density of patients with cerebral palsy. Dev Med Child Neurol 1992; 34 (suppl): 38

9 Dent CE, Richens A, Rowe DJF, Stamp TCB Osteomalacia with long-term anticonvulsant therapy in epilepsy. $B M$ F 1970; iv: 69-72.

$10 \mathrm{Hahn}$ TJ. Drug-induced disorders of vitamin $\mathrm{D}$ and mineral metabolism. Clinical Endocrinology and Metabolism 1980; 9: $107-29$.

11 Kruse $\mathrm{K}$. On the pathogenesis of anticonvulsant druginduced alterations of calcium metabolism. Eur $₹$ Pediatr 1982; 138: 202-5.

12 Storm T, Thamsborg G, Steiniche T, Genant HK, Sorensen $\mathrm{OH}$. Effect of intermittent cyclical etidronate therapy on bone mass and fracture rate in women with postmenopausal osteoporosis. N Engl f Med 1990; 322: 1265-71.

13 McCloskey EV, Yates AJP, Gray RES, Hamdy NAT, Galloway J, Kanis JA. Diphosphonates and phosphate homeostasis in man. Clin Sci 1988; 74: 607-12.

14 Nagant de Deuxchaisnes C, Rombouts-Lindemans C, Huaux JP, Devogelaer JP. Diphosphonates and inhibition of bone mineralisation. Lancet 1982; ii: 607-8. 